Holger Görg*

\title{
Ausländische Direktinvestitionen in Deutschland - die große Angst vor China
}

https://doi.org/10.1515/zfwp-2019-2004

Abstract: The current discussion in German policy circles suggests a strong scepticism towards foreign takeovers of German firms. This essay takes issue with this. It is firstly argued that Germany in actual fact receives relatively little inward investment. The essay then discusses the potential benefits from inward FDI which could also benefit the German economy. It then briefly reflects on the German policy initiatives which seem to be based on a fear of investment from China - though it is not clear at all what this fear is based on.

\section{Einleitung}

Die Novellierung der deutschen Außenwirtschaftsverordnung und die vor kurzem von Bundesminister Peter Altmaier vorgestellte Nationale Industriestrategie 2030 deuten auf eine Grundhaltung in der Politik hin, die ausländischen Direktinvestitionen in Deutschland, insbesondere durch Übernahmen deutscher Unternehmen, erst einmal skeptisch gegenübersteht. Es wird oft von einem „Ausverkauf“ deutscher Unternehmen an ausländische Eigner gesprochen. Ganz besonders skeptisch scheint man gegenüber chinesischen Investoren zu sein.

Dieser kurze Aufsatz steht dieser Grundhaltung kritisch gegenüber. Zuerst wird gezeigt, dass ausländische Investitionen in Deutschland relativ gering sind, sowohl im Vergleich zu anderen Ländern als auch im Vergleich zu Investitionen deutscher Unternehmen im Ausland. Dann wird die Literatur diskutiert die klar zeigt, dass ausländische Unternehmen in Gastland in vielen Fällen positive Effekte auf das Gastland haben. Es gibt hier keine Evidenz, dass sich chinesische Unternehmen anders verhalten als solche aus anderen Ländern. Schließlich wird darüber spekuliert, warum chinesische Investoren als besonders „gefährlich“ in der Öffentlichkeit und Politik angesehen werden.

\footnotetext{
*Kontakt: Holger Görg, Kiel Centre for Globalization, Institut für Weltwirtschaft, Kiellinie 66, 24105 Kiel, Tel.: (0431) 8814 258, E-Mail: holger.goerg@ifw-kiel.de 


\section{Ausländische Direktinvestitionen in Deutschland - eine Bestandsaufnahme}

Der Bestand ausländischer Direktinvestitionen - also Investitionen ausländischer multinationaler Unternehmen - in Deutschland lag 2017 bei rund 931 Milliarden US Dollar (UN, 2018). Das hört sich erst einmal nach viel an, entspricht aber gemessen an den weltweiten Direktinvestitionen nur knapp unter 3\%. Zum Vergleich: Deutschlands Anteil am Weltbruttoinlandsprodukt liegt bei rund 4,5\%. ${ }^{1}$ Damit erreicht Deutschland bei den eingehenden Direktinvestitionen einen Wert vergleichbar mit Frankreich - aber auch mit den viel kleineren Volkswirtschaften der Niederlande, Irlands oder etwa Kanadas. Im Gegensatz dazu erreicht Großbritannien einen Wert von rund 5\%, China von $8 \%$ und die USA von $25 \%$ (7,8 Billionen US Dollar) der weltweiten Direktinvestitionen.

Im Vergleich dazu sind die deutschen Direktinvestitionen im Ausland mit 1,61 Billionen US Dollar im Jahr 2017 um rund dreiviertel höher als die Direktinvestitionen in Deutschland. Eine ähnliche Diskrepanz zwischen ein- und ausgehenden Direktinvestitionen ist auch bei Ländern wie Frankreich oder den Niederlanden zu sehen, wohingegen Großbritannien, Irland, die USA und China eine viel ausgewogenere Balance aufzeigen.

Zusammengenommen könnten diese zwei Fakten - Deutschlands relativ geringer Anteil an weltweiten eingehenden Direktinvestitionen und seine viel höheren ausgehenden Direktinvestitionen - durchaus auf eine mangelnde Attraktivität des Standort Deutschlands hinweisen. Deutschland scheint also für ausländische Konzerne nicht die erste Adresse zu sein, wenn es um internationale Standortentscheidungen geht.

Das kann viele Gründe haben: Die Literatur zu den Determinanten ausländischer Direktinvestitionen zeigt, dass Lohn- und Lohnnebenkosten, ebenso Unternehmenssteuern, im potentiellen Gastland eine wichtige Rolle spielen (Blonigen, 2005; Campos und Kinoshita, 2003). Bei beiden Aspekten liegt Deutschland nicht gerade im niedrigen Bereich. Außerdem gibt es für Unternehmen aus benachbarten EU-Ländern keine Notwendigkeit, in Deutschland zu investieren, da sie problemlos exportieren und importieren können. Das sogenannte „tariff jumping FDI“ (Blonigen, 2005) spielt also keine besonders große Rolle. Nicht zuletzt erschweren bürokratische oder institutionelle Hürden im Gastland generell das Engagement ausländischer Unternehmen (Blonigen, 2005). Im „Ease of doing business“-Ranking der Weltbank - nur einer von vielen Versuchen, solche

1 Eigene Berechnung basierend auf Daten der Weltbank https://data.worldbank.org/ (zugegriffen am 13.03.2019) 
Hürden zu quantifizieren - liegt Deutschland aktuell nur auf Platz $24 .^{2}$ Nicht nur hinter den USA, Großbritannien, Kanada und Irland, sondern auch z. B. Neuseeland, Südkorea, Estland, Lettland und den skandinavischen Ländern. Auch das kann sich negativ auf die internationale Wahrnehmung der Qualität des Standorts Deutschlands auswirken.

\section{Ausländische Direktinvestitionen können produktivitätssteigernd wirken}

Dabei können ausländische Direktinvestitionen ein wichtiger Treibstoff für den Motor einer Volkswirtschaft sein. Sie bringen nicht nur zusätzliches Kapital, sondern auch neue Technologie und sorgen außerdem für höheren Wettbewerb. Dies kann positive Wachstumseffekte haben, die über die Effekte einheimischen Kapitals hinausgehen können (Borensztein et al., 1998, Görg und Greenaway, 2004).

Die moderne mikroökonometrische Außenhandelsforschung zeigt, basierend auf der Analyse von großen Datensätzen auf Unternehmensebene, dass ausländische Unternehmen im Gastland im Durchschnitt produktiver sind als vergleichbare einheimische Unternehmen. Dies ist nicht nur darauf zurückzuführen, dass ausländische Investoren hochproduktive Firmen aufkaufen, sondern auch darauf, dass diese Unternehmen nach der Übernahme ihre Produktivität steigern. Dies gilt nicht nur für Entwicklungs- und Schwellenländer, sondern auch für hochindustrialisierte Volkswirtschaften wie die USA, Großbritannien und Deutschland (Criscuolo und Martin, 2009; Temouri et al., 2008). Dieser Produktivitätsvorteil wird generell dadurch erklärt, dass ausländische Unternehmen einen Technologievorsprung gegenüber einheimischen Wettbewerbern haben, den sie mit ins Gastland bringen. Diesen brauchen sie, um im Wettbewerb im Gastland bestehen zu können und den intrinsischen Nachteil des „ausländisch seins“ (d.h. höhere Kosten für z. B. Transport und Kommunikation) wett zu machen.

Liegt ein solcher Technologievorsprung vor, gibt es auch Potential für sogenannte „Spillover-Effekte“ auf benachbarte einheimische Unternehmen. Diese können die Technologie, die in multinationalen Unternehmen angewendet wird, lernen, z. B. durch Imitation, Abwanderung von Beschäftigten von ausländischen zu einheimischen Firmen, und Zuliefer- oder Kundenbeziehungen zwischen multinationalen und einheimischen Firmen. Zudem setzt der zusätzliche

2 Siehe http://www.doingbusiness.org/en/rankings (zugegriffen am 22. März 2019). 
Wettbewerbsdruck durch ausländische Konkurrenten Anreize für einheimische Unternehmen, ihre Produktionstechnologie und Produktivität zu verbessern. Dieser verstärkte Konkurrenzdruck kann jedoch auch negative Effekte für einheimische Firmen mit sich bringen, die diesem Druck nicht standhalten können. Görg und Marchal (2019), Haskel et al. (2005) und Keller und Yeaple (2009) zeigen mit französischen, britischen bzw. US amerikanischen Mikrodaten, dass ausländische Direktinvestitionen in den drei Ländern im Durchschnitt positive Spillover-Effekte auf das Produktivitätswachstum einheimischer Unternehmen haben. Vergleichbare Studien für Deutschland sind leider nicht verfügbar. Da es sich jedoch um eine ähnlich hoch-industrialisierte Volkswirtschaft handelt, spricht vieles dafür, hier von ähnlichen Effekten auszugehen.

Weiterhin zahlen ausländische Unternehmen im Gastland im Durchschnitt auch meist höhere Löhne als vergleichbare lokale Wettbewerber. Dies zeigen z. B. Girma und Görg (2007) und Hijzen et al. (2013) mit Daten für Großbritannien, Deutschland und Brasilien. Dies kann vor allem darauf zurückgeführt werden, dass ausländische Unternehmen neue hochqualifizierte Arbeitsplätze schaffen, die mit höheren Löhnen einhergehen.

Deshalb sollte es gerade angesichts der anhaltenden Schwäche des Produktivitätswachstums in Deutschland für die Wirtschaftspolitik Priorität haben, die Attraktivität des „Standort Deutschlands“ für ausländische Unternehmen zu steigern. Weltweit setzen in der Tat viele Länder aktiv Politikmaßnahmen ein, um ausländische Direktinvestitionen ins Land zu ziehen. Diese müssen nicht immer finanzieller Natur (sprich Steuern oder Subventionen) sein, sondern können oder sollten eher aus weicheren Maßnahmen, wie der Bereitstellung von Informationen und Infrastruktur, oder der Signalisierung von Offenheit, z. B. durch Investitionsfördergesellschaften (Investment Promotion Agencies) bestehen (Moran et al., 2017).

\section{Deutschland - hin zum Protektionismus?}

In Deutschland (und anderen EU Partnerländern) wird jedoch gerade scheinbar über das Gegenteil nachgedacht. Die Außenwirtschaftsverordnung soll novelliert werden, um damit die Beteiligungsschwelle zu senken, ab der die Bundesregierung einen Einstieg von Nicht-EU Investoren prüfen und gegebenenfalls untersagen kann. Zwar beträfe das zunächst nur relativ wenige Wirtschaftsbereiche und Investoren in Hochtechnologiebranchen unmittelbar. Doch schon das Signal, dass nationale Interessen und Protektionismus Gewicht gewinnen, kann Deutschland in den Augen von Investoren weniger attraktiv erscheinen lassen. 
Verteidiger der neuen Regeln verweisen darauf, dass es nur um Fälle gehe, in denen die „öffentliche Sicherheit und Ordnung“ gefährdet sind - aber wie die Trump-Regierung vor kurzem bei der Einführung von Straffzöllen auf Stahl und Aluminium gezeigt hat, ist das ein sehr dehnbares Konzept, das für protektionistische Maßnahmen missbraucht werden kann. So etwas schreckt Investoren ab.

Die vor kurzem von Herrn Bundesminister Peter Altmaier vorgestellte Nationale Industriestrategie 2030 nimmt dieses Thema ebenfalls auf. Zwar sollen staatliche Eingriffe bei ausländischen Übernahmen, wie in der AWV auch, auf den Bereich der Nationalen Sicherheit beschränkt werden. Das Papier legt aber auch dar, dass bei Übernahmen in Bereichen der „Technologie- und Innovationsführerschaft“ der Staat nur in Ausnahmefällen einschreiten sollte, es aber vorrangig Aufgabe der Privatwirtschaft sei, diese Übernahmen durch ausländische Wettbewerber zu „verhindern“. Die Wahl des Wortes „verhindern“ zeigt unmissverständlich, dass solche ausländischen Übernahmen nicht erwünscht sind. Und dieses negative Signal an ausländische Investoren wird durch die Novellierung der AWV nur verstärkt.

\section{China - die große Gefahr?}

In der öffentlichen Diskussion geht es bei diesen möglichen Verschärfungen im Umgang mit ausländischen Investoren augenscheinlich vor allem um Investitionen aus China. Diesen wird unterstellt, sie wollten nur neue Technologien im Gastland (sprich Deutschland) erlernen und diese dann nach China transferieren. Worauf diese Unterstellung basiert, ist meist nicht klar. Der deutsche Roboterhersteller Kuka wird zwar gerne als Negativbeispiel aufgeführt, aber selbst hier ist bei näherem Hinschauen nicht erkennbar, dass chinesische Investoren nur am Absaugen der Technologie interessiert sind. Und Studien, die mit belastbaren empirischen Analysen diese Furcht vor chinesischen Investoren untermauern, fehlen komplett. Das ist nicht verwunderlich, gab es doch, laut Bundesbank Angaben, 2016 nur 139 Unternehmen mit chinesischer Beteiligung in Deutschland. Zum Vergleich: es gab 120 Unternehmen mit irischen Eigentümern und 1380 US-amerikanische Betriebe in Deutschland. Und 2161 deutsche Unternehmen hatten Niederlassungen in China. ${ }^{3}$

Unter diesen Unternehmen mag es bestimmt schwarze Schafe geben. Sie müssen im internationalen Wettbewerb bestehen und für einige mag dies bein-

3 Daten von https://www.bundesbank.de/de/statistiken/aussenwirtschaft/direktinvestitionen/-/ bestandsangaben-ueber-direktinvestitionen-739416 (zugegriffen am 22. März 2019). 
halten, Wirtschaftsspionage zu betreiben oder ausländische Konkurrenten auszuschalten, um ihre Technologie und Wettbewerbsposition zu verbessern. Das gilt jedoch für Unternehmen aller Herkunftsländer. Doch die Evidenz, dass im Durchschnitt positive Effekte aufs Gastland auftreten (siehe oben), besteht. Und bis zum Vorliegen belastbarer Evidenz, die das Gegenteil zeigt, sollte davon ausgegangen werden, dass dies auch für Investitionen aus China zutrifft. Seien es neue Investitionsmöglichkeiten, die Erschließung neuer Märkte (was allem Anschein nach für Kuka eine wichtige Rolle spielte), neue Möglichkeiten der Finanzierung, neue Managementmethoden, oder Technologie: all dies sind Beispiele für Vorteile, die ausländische Eigner mitbringen können.

Die oben erwähnte Studie von Görg und Marchal (2019) zeigt in ihrer Analyse für Frankreich, dass von ausländischen Investoren übernommene Unternehmen ihre Produktivität nach der Übernahme im Durchschnitt erhöhen. In einem Vergleich deutscher mit anderen EU und nicht-EU Übernahmen zeigen sich keine Unterschiede in diesem Ergebnis. Letztere beinhalten auch chinesische Übernahmen. Diese konnten jedoch auch hier wegen der geringen Fallzahl nicht separat untersucht werden.

Und nicht zu vergessen: Auch wenn deutsche Unternehmen zum Beispiel in den USA investieren kommt es zum Transfer von Technologien und Methoden ins Heimatland. Dies ist ein Zeichen der internationalen Verflechtungen der Wirtschaft, von denen gerade Deutschland in den letzten Jahrzehnten stark profitiert hat.

Woher stammt also die Angst vor chinesischen Investoren? In den Debatten wird immer wieder darauf hingewiesen, dass viele chinesische Unternehmen zumindest teilweise in staatlicher Eigentümerschaft sind. Und dass sie damit automatisch als Spione einer autokratischen chinesischen Regierung operieren. Die chinesische Regierung hat gewiss nicht dasselbe Demokratieverständnis wie, z. B. die deutsche. Aber daraus kann nicht geschlossen werden, dass chinesische Investoren nur Handlanger der Regierung sind. Historisch gesehen ist ein Hauptgrund für die Existenz staatlicher Unternehmen in China die Schaffung und der Erhalt von Arbeitsplätzen, was auch damit einhergeht, dass diese Unternehmen generell weniger produktiv und profitabel sind als private (vgl. Eckaus, 2006). Entsprechend ist die Bedeutung staatlicher Unternehmen in China in den letzten Jahrzehnten rapide gefallen. ${ }^{4}$ Auch hier fehlt die belastbare Evidenz, um die Angst vor chinesischen Investoren zu untermauern.

4 Im Jahr 2009 hielten staatliche Unternehmen etwa $8 \%$ des gesamten Kapitalstocks im Industriesektor (Chang und Jin, 2016). 


\section{Schlussbetrachtung}

Die in der Nationalen Industriestrategie 2030 und in der Novellierung der Außenwirtschaftsverordnung zumindest unterschwellig mitschwingende Skepsis (oder Angst?) gegenüber ausländischen Investitionen - insbesondere aus China - entbehrt jeder empirischen Grundlage. Investitionen in Unternehmen, die relevant für die „Nationale Sicherheit“ sind, sollten natürlich kritisch geprüft werden können - wobei jedoch eine sehr enge Definition der „Nationalen Sicherheit“ als „verteidigungsrelevant“ glaubhaft vorgenommen werden sollte. Das Hauptkriterium im Umgang mit ausländischen Investoren sollte aber eher „Offenheit“ sein. Deutschland kann durchaus mehr ausländische Investitionen vertragen und diese können im Durchschnitt produktivitätssteigernd sein. Technologietransfer wird dann natürlich stattfinden, aber dieser geht in beide Richtungen und kann sich positiv sowohl auf das Gastland (sprich Deutschland) als auch auf das Heimatland der Investoren auswirken.

\section{Literatur}

Borensztein, E., J. De Gregorio und J.-W. Lee (1998), How does foreign direct investment affect economic growth?, Journal of International Economics 45(1), S. 115-35.

Blonigen, Bruce A., 2005, „A Review of the Empirical Literature on FDI Determinants.“ Atlantic Economic Journal 33, S. 383-403.

Campos, N. und Y. Kinoshita, 2003, Why Does FDI Go Where it Goes? New Evidence from the Transition Economies, IMF Working Paper No. 03/228

Chang, S.J. und S.Y. Jin, 2016, The performance of state-owned enterprises in China: An empirical analysis of ownership control through SASACs, Centre for Governance, Institutions and Organisations (CGIO), NUS Business School National University of Singapore (https://bschool.nus.edu.sg/Portals/0/docs/CGIO/soe-china-researchreport-2016.pdf)

Criscuolo C., R. Martin (2009). Multinationals and U.S. Productivity Leadership: Evidence from Great Britain. Review of Economics and Statistics 91(2), S. 263-281.

Eckaus, R.S. (2006), China's exports, subsidies to state owned enterprises and the WTO, China Economic Review 17, S. 1-13.

Girma, S. und H. Görg (2007), Evaluating the foreign ownership wage premium using a differencein-differences matching approach, Journal of International Economics 72(1), S. 97-112.

Görg, H., D. Greenaway (2004). Much ado about nothing? Do domestic firms really benefit from foreign direct investment?, World Bank Research Observer 19(2), S. 171-197.

Görg, H. und L. Marchal, 2019, Die Effekte deutscher Direktinvestitionen im Empfängerland vor dem Hintergrund des Leistungsbilanzüberschusses: Empirische Evidenz mit Mikrodaten für Frankreich, Perspektiven der Wirtschaftspolitik, im Erscheinen.

Haskel, J. E., S. Pereira und M. Slaughter (2007), Does inward foreign direct investment boost the productivity of domestic firms?, Review of Economics and Statistics 89, S. 482-96. 
Hijzen, A., P. Martins, T. Schank und R. Upward (2013), „Foreign-owned firms around the world: a comparative analysis of wages and employment at the micro-level“, European Economic Review 60, S. 170-188.

Keller, W. und S. R. Yeaple (2009), Multinational enterprises, international trade, and productivity growth: Firm-level evidence from the United States, Review of Economics and Statistics 91(4), S. 821-31.

Moran, T., H. Görg, A. Seric und C. Krieger-Boden (2017), How to attract Quality FDI?, KCG Policy Paper No. 2, Kiel Centre for Globalization.

Temouri, Y., N. Driffield und A. Higón, D. (2008). Analysis of productivity differences among foreign and domestic firms: evidence from Germany. Review of World Economics 144(1), S. 32-54.

UN (2018), World Investment Report 2018, Genf, United Nations. 